\title{
Effect of Selenium Supplementation on Recurrent Hyperthyroidism Caused by Graves' Disease: A Prospective Pilot Study
}

Authors

Affiliations

\section{Wang ${ }^{1{ }^{*}}$, B. Wang ${ }^{1{ }^{*}}$, S. R. Chen ${ }^{2 *}$, X. Hou ${ }^{1}$, X. F. Wang ${ }^{3}$, S. H. Zhao ${ }^{1}$, J. Q. Song ${ }^{4}$, Y. G. Wang}

Department of Endocrinology, the Affiliated Hospital of Qingdao University, Qingdao, China Department of Science and Technology, Jinzhou Medical University, Jinzhou, China Department of Nuclear Medicine, the Affiliated Hospital of Qingdao University, Qingdao, China Department of Nursing, Yeda Hospital of Yantai City, Yantai, China
Key words

selenium

- hyperthyroidism

- Graves' disease

received 07.03.2016 accepted 06.06.2016

Bibliography DOI http://dx.doi.org/ 10.1055/s-0042-110491 Published online: July 8, 2016

Horm Metab Res 2016; 48: 559-564

(c) Georg Thieme Verlag KG Stuttgart · New York ISSN 0018-5043

Correspondence

\section{Y. G. Wang, MD}

Department of Endocrinology The Affiliated Hospital of Qingdao University

16 Jiangsu Road

Qingdao 266003

China

Tel.: + 86/0532/82911740

Fax: + 86/0532/82911 740

bennettzhwang@gmail.com

\section{J. Q. Song}

Department of Nursing Yeda Hospital of Yantai City Yantai 264006

China

songjqytyd@163.com

\section{Abstract}

$\nabla$

The effect of selenium supplementation on recurrent hyperthyroidism caused by Graves' disease is unclear. Our study aimed to assess the efficacy of selenium supplementation therapy on recurrent Graves' disease. Forty-one patients with recurrent Graves' disease were enrolled in this study. All patients received the routine treatment using methimazole (MMI), while patients allocated to the selenium group received additional selenium therapy for 6 months. The influence of selenium supplementation on the concentrations of thyroid stimulating hormone (TSH), anti-TSH-receptor antibodies (TRAb), free thyroxine (FT4), and free triiodothyronine (FT3) were assessed. The remission rate was also compared between 2 groups. There was no obvious difference in the demographic data and the levels of serum FT4, FT3, TSH, and TRAb between the 2

\section{Introduction}

\section{$\nabla$}

Graves' disease is the major cause of hyperthyroidism, and nearly $3 \%$ of women and $0.5 \%$ of men suffer from Graves' disease [1]. In Graves' disease, anti-TSH-receptor antibodies (TRAb) bind to thyroid-stimulating hormone (TSH) receptor and stimulate the production of thyroid hormones and thyroid hypertrophy $[2,3]$. Antithyroid drugs (ATDs) inhibiting thyroid hormone syntheses are the principal treatment method for Graves' disease, but it is associated with high rate of recurrence. About $50 \%$ patients will achieve remission after receiving ATDs therapy for about $12-18$ months [4-6], but there is high prevalence of recurrence in those patients with remission, ranging from 30 to $70 \%$ in published studies [7-9]. Some scholars recommend

${ }^{*}$ L. Wang, B. Wang, and S. R. Chen contributed equally to the study groups at baseline. Both FT4 and FT3 decreased more at 2 months in the selenium group than the controls, while the TSH level increased more in patients receiving selenium supplementation $(\mathrm{p}<0.05)$. The TRAb level was significantly lower in patients receiving selenium supplementation (2.4 IU/l vs. 5.6 IU/l, p=0.04). The percentages of patients with normal TRAb level at 6 months was also significantly higher in the selenium group ( 19.0 vs. $0 \%, p=0.016$ ). Kaplan-Meier survival curve showed patients receiving selenium supplementation had a significantly higher rate of remission than controls (Log-rank test $\mathrm{p}=0.008$ ). In conclusion, selenium supplementation can enhance the effect of antithyroid drugs in patients with recurrent Graves' disease. Randomized trials with large number of participants are needed to validate the finding above.

Supporting Information for this article is available online at http://www.thieme-connect.de/products

radioactive iodine or thyroidectomy to patients with recurrent Graves' disease, since a second course of ATDs therapy may have little chance to lead to successful remission. However, it is still unclear which is the best treatment strategy for patients with recurrent Graves' disease, and more studies are urgently needed to explore more new promising treatment strategy for recurrent hyperthyroidism $[4,10]$.

Selenium is intensively involved in the immune response, and it also has important roles in maintaining the normal function of thyroid $[11,12]$. Selenium is an essential particle in the active site of some enzymes in the thyroid, such as glutathione peroxidases (GPXs) and thioredoxin reductases $[13,14]$. Selenium can protect thyrocytes against the damage from free radicals and oxidative stress [15-17]. Sufficient selenium status in the body is necessary for maintaining the normal function of thyroid, while its deficiency 
can result in decreased activity of GPXs and oxidative damage in the thyroid. Moreover, selenium deficiency can cause impaired immune tolerance and autoimmune processes in the thyroid gland, and thus is involved in the pathogenesis of autoimmune thyroiditis or Graves' disease $[18,19]$. Previous studies have found that selenium deficiency is common in patients with Graves' disease and serum selenium concentrations are correlated with thyroid hormones in both hyperthyroidism patients and healthy controls [20-22]. Previous studies have identified that selenium supplementation has some efficacy on Graves' orbitopathy [23], but the efficacy of selenium supplementation on recurrent Graves' disease has not been studied. Our study aimed to assess the efficacy of selenium therapy on recurrent hyperthyroidism caused by Graves' disease.

\section{Subjects and Methods \\ $\nabla$}

\section{Participants and treatment}

In this prospective pilot study, we consecutively enrolled patients with recurrent Graves' disease as study subjects, who were undergoing ATDs treatment from September 2013 through June 2014 at our hospital. Graves' disease was diagnosed according to the guideline from the American Thyroid Association. All patients were suffering from recurrent Graves' disease, and had a history of ATDs use for at least 2 years. Recurrent Graves' disease was diagnosed by the clinical features of Graves' disease recurrence including the presence of hyperthyroidism symptoms, increased levels of free thyroxine (FT4) and free triiodothyronine (FT3), decreased level of TSH, and a history of hyperthyroidism remission after a finished regular ATDs therapy. Patients were excluded if they were pregnant women or prepared for pregnancy. Patients treated with radioiodine therapy or thyroidectomy were also excluded. Forty-one patients with recurrent Graves' disease were finally enrolled in this study. Twenty-one patients were allocated to the combination therapy group and received additional selenium supplementation for 6 months, while all patients received a standard antithyroid treatment with methimazole (MMI). The method of allocating participants was a quasi-random method, which mainly allocated patients by date of consultation. This study was approved by the ethical committee in our hospital, and informed consent was obtained from all included patients. We used sodium selenite for the selenium supplementation with a therapy strategy of $100 \mu \mathrm{g} 2 \times / \mathrm{d}$ for 6 months, which was also used in previously published studies $[23,24]$. All patients were followed at least 18 months to observe the remission rate.

\section{Data collection and endpoints}

Parameters of thyroid function used in this study included TSH, TRAb, FT4, and FT3. Patients were scheduled to re-check at 1 month, 2 months, and 3 months after initiating treatment. For patients with normal levels of both FT4 and FT3 at 3-month visit, they were scheduled to re-check every 2 or 3 months. Serum TRAb values were determined by assay every 3 months, and the levels of FT4, FT3, and TSH were determined by assay on every visit.

The primary endpoint was the effect of selenium supplementation on the concentrations of TSH, TRAb, FT4, and FT3 during follow-up. The second endpoint was the remission rate. Remission was defined as keeping euthyroid with or without receiving low-dose MMI (e.g., $5 \mathrm{mg} / \mathrm{d}$ ) for at least 12 months. Those patients remaining euthyroid and having normal level of TRAb for at least 6 months were also considered to have achieved remission. The difference between the 2 groups with regard to the dose of MMI therapy was also assessed.

\section{Statistical analysis}

Parameters in normal distributions were presented as mean (SD), while those without normal distributions were presented as median values [interquartile range (IQR)]. Parameters in normal distributions were analyzed using 2-sided $t$-test. MannWhitney U-test was used for data not in normal distributions. Categorical variables were analyzed using the $\mathrm{x}^{2}$ test or Fisher's exact test. The effect of selenium supplementation on remission was further evaluated using Kaplan-Meier survival analysis. The STATA program was used, and significance was defined as $\mathrm{p}<0.05$.

\section{Results \\ $\nabla$ \\ Baseline characteristics}

There were 21 patients in the selenium group (16 females, $76.2 \%$ ) and 20 patients (18 females, $90.0 \%$ ) in the control group ( $\odot$ Table 1). The mean age of Selenium group and controls were 37.4 years and 38.9 years, respectively ( $\Theta$ Table 1 ). Two patients were lost during follow-up in the selenium group, while one patient was lost during follow-up in the controls. Seventeen (80.9\%) patients revisited regularly in the selenium group and $18(90.0 \%)$ patients revisited regularly in the controls group. There was no statistical difference in gender, age, and the levels of serum FT4, FT3, TSH, and TRAb between the 2 groups at baseline ( $\odot$ Table 1). The median time of follow-up on was 19.5 months, ranging from 12 to 26 months. At baseline, there was no obvious difference between the 2 groups with regard to the dose of MMI therapy $(17.8 \mathrm{mg} / \mathrm{d}$ vs. $18.5 \mathrm{mg} / \mathrm{d} ; \mathrm{p}=0.78)$.

Table 1 Demographic and hormonal data of patients at inclusion by groups.

\begin{tabular}{|c|c|}
\hline Characteristics & Selenium group $(\mathbf{n}=\mathbf{2}$ \\
\hline Mean age (years) & $37.4 \pm 15.0$ \\
\hline No. of females (\%) & $16(76.2 \%)$ \\
\hline TSH $(\mathrm{mlU} / \mathrm{l})^{*}$ & $0.005(0.005-0.011)$ \\
\hline $\mathrm{FT} 4(\mathrm{pmol} / \mathrm{l})^{*}$ & $31.6(27.1-40.2)$ \\
\hline FT3 $(\mathrm{pmol} / \mathrm{l})^{*}$ & $13.1(9.9-19.9)$ \\
\hline $\operatorname{TRAb}(I U / I)^{*}$ & $10.2(5.6-18.3)$ \\
\hline No. of patients revisiting regularly (\%) & $17(80.9 \%)$ \\
\hline MMI dose $(\mathrm{mg} / \mathrm{d})$ & $17.8 \pm 7.2$ \\
\hline No. of dropouts (\%) & $2(9.5 \%)$ \\
\hline
\end{tabular}

Selenium group $(\mathbf{n}=\mathbf{2 1})$

$0.005(0.005-0.011)$

$0.2(5.6-18.3)$

$\begin{array}{ll}\text { Control group }(\mathbf{n = 2 0}) & \mathbf{p} \text {-Value } \\ 38.9 \pm 14.3 & 0.28 \\ 18(90.0 \%) & 0.23 \\ 0.005(0.005-0.011) & 0.97 \\ 26.8(23.6-34.1) & 0.27 \\ 11.1(8.3-13.8) & 0.32 \\ 12.4(8.3-15.3) & 0.69 \\ 18(90.0 \%) & 0.41 \\ 18.5 \pm 7.4 & 0.78 \\ 1(5.0 \%) & 0.57\end{array}$




\section{Comparison of thyroid function and MIMI therapy}

The concentrations of FT4 and FT3 decreased faster in the selenium supplementation group ( $\bullet$ Fig. 1). The FT3 concentration at 2 months was obviously lower in those patients allocated to selenium group ( $5.4 \mathrm{pmol} / \mathrm{l} \mathrm{vs.} 7.3 \mathrm{pmol} / \mathrm{l}, \mathrm{p}=0.009$ ), and the FT4 concentration at 2 months was also significantly lower in those patients receiving selenium therapy $(14.9 \mathrm{pmol} / \mathrm{l} \mathrm{vs.} 18.4 \mathrm{pmol} / \mathrm{l}$, $\mathrm{p}=0.047)($ Table 1S, $\odot$ Fig. 1). The TSH level at 2 months was significantly higher in patients receiving selenium supplementation $(0.023 \mathrm{mIU} / \mathrm{l}$ vs. $0.007 \mathrm{mIU} / \mathrm{l}, \mathrm{p}=0.01)$ (Table 1S, ○ Fig. 1 ). The FT4 concentration at the final follow-up was obviously lower in the selenium group $(15.8 \mathrm{pmol} / \mathrm{l}$ vs. $18.8 \mathrm{pmol} / \mathrm{l}$, $\mathrm{p}=0.02$ ), and the TRAb level was also significantly lower in those patients receiving selenium therapy at the final follow-up (2.4 IU/l vs. 5.6IU/l, p=0.04) (Table 1S, ○ Fig. 1).

The percentage of patients with normalizing level of FT4 at 2-month visit was significantly higher in those patients receiv- ing selenium therapy ( 85.7 vs. $65.0 \%, \mathrm{p}=0.03$ ) ( $\bullet$ Fig. 2 ). Patients with normalizing level of FT3 at 2-month visit were more common in those patients receiving selenium therapy (80.9 vs. $55.0 \%)$, though there was no statistical significance in the difference $(p=0.07)(\theta$ Fig. 2$)$. The percentage of patients with normal TRAb level at 6-month visit was also significantly higher in the selenium group ( 19.0 vs. $0 \%, p=0.016)$. The percentage of patients with normal TRAb level at the final follow-up was $33.3 \%$ in the selenium group, while this percentage in the controls was $10.0 \%(\mathrm{p}=0.06)$ (० Fig. 2 ).

The dose of MMI therapy in the selenium group was lower than that in the control group at 6 -month follow-up $(8.0 \mathrm{mg} / \mathrm{d}$ vs. $15.0 \mathrm{mg} / \mathrm{d} ; \mathrm{p}=0.001)$ and at the last follow-up $(5.9 \mathrm{mg} / \mathrm{d}$ vs. $11.1 \mathrm{mg} / \mathrm{d} ; \mathrm{p}=0.009$ ). The frequency of patients stopping MMI therapy tended to be lower in the selenium group than that in the control group (33.3 vs. $10.0 \%, \mathrm{p}=0.09$ ) (Table 1S).

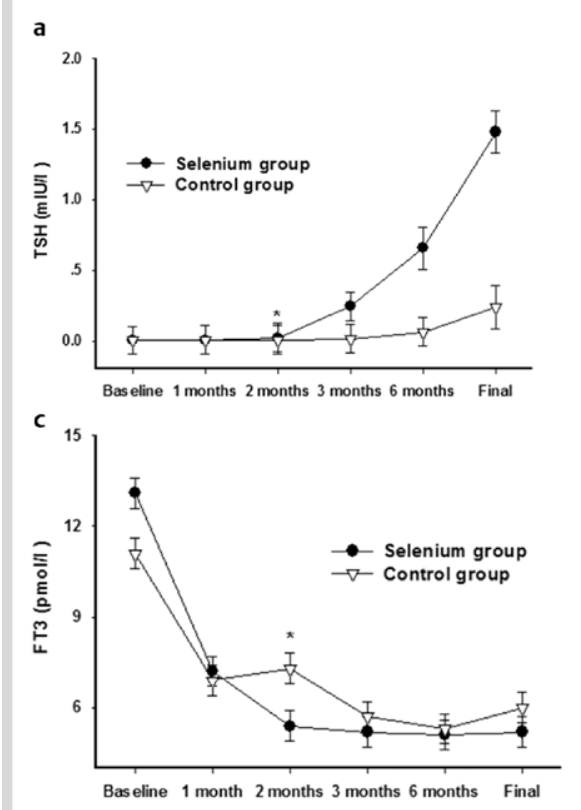

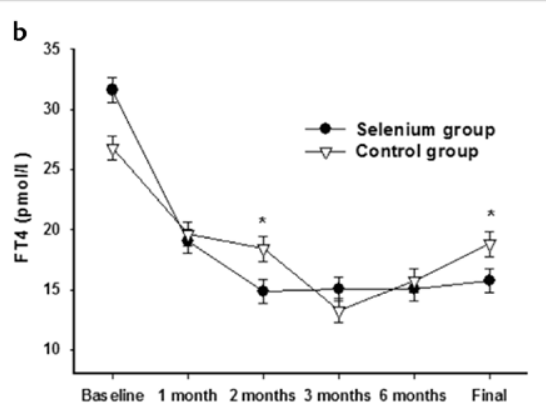

d

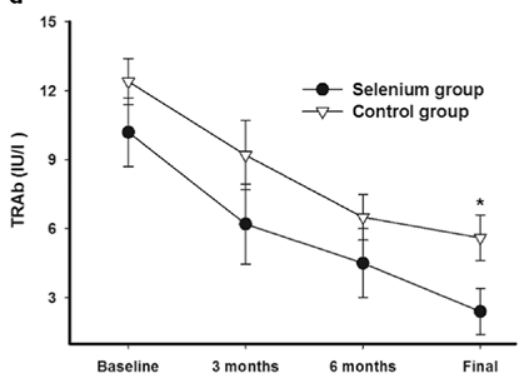

Fig. 1 Effect of selenium supplementation in normalizing serum levels of TSH, FT3, FT4, and TRAb in patients with recurrent Graves' disease.
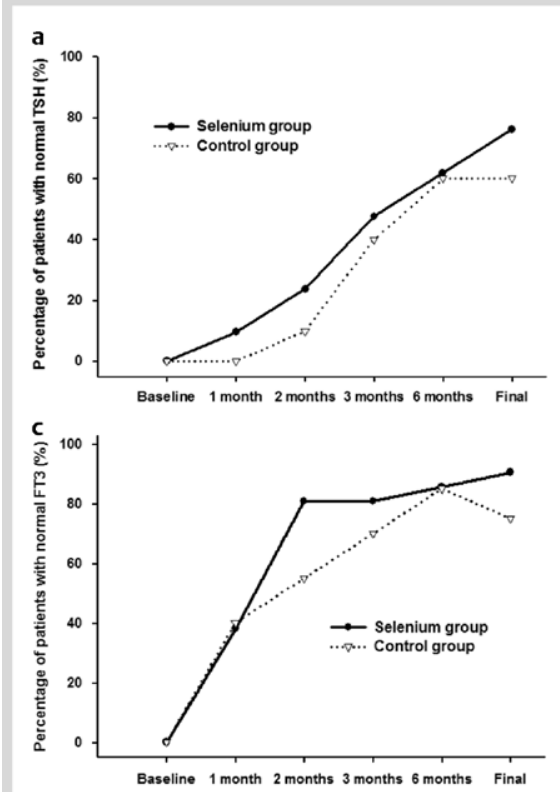
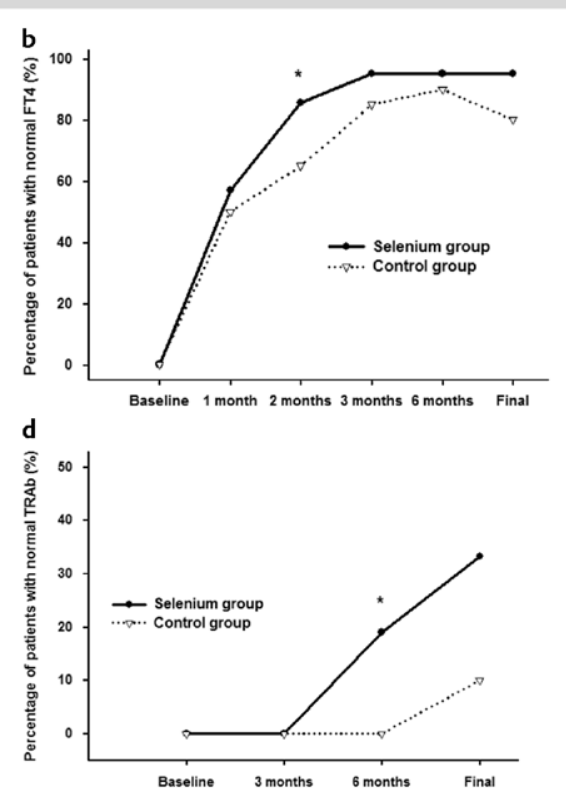

Fig. 2 Effect of selenium supplementation on the percentages of patients with normal TSH, normal $\mathrm{FT} 3$, normal FT4, and normal TRAb in recurrent Graves' disease. 


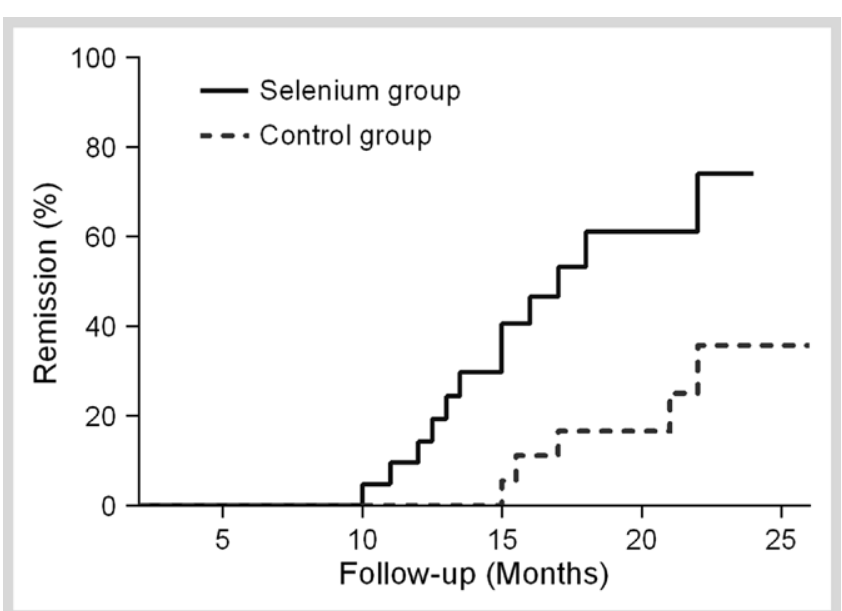

Fig. 3 Kaplan-Meier survival curve showing the cumulative rate of remission by groups in patient with recurrent Graves' disease (Log-rank test $\mathrm{p}=0.008$ ).

\section{Comparison of remission rate}

There were 11 (52.3\%) patients attaining remission at the final follow-up in the selenium supplementation group, and the number of patients attaining remission in the control group was $5(25.0 \%)$. There was a tendency of higher remission rate in the selenium supplementation group ( 52.3 vs. $25.0 \%, \mathrm{p}=0.07$ ). Kaplan-Meier survival curve showed patients receiving selenium supplementation had a significantly higher rate of remission than controls (Log-rank test $\mathrm{p}=0.008)$ ( $\mathrm{O}$ Fig. $\mathbf{3}$ ).

\section{Discussion}

$\nabla$

This study assessed the effect of selenium supplementation on recurrent hyperthyroidism caused by Graves' disease. Both FT4 and FT3 decreased more at 2 months in the selenium group than the controls, while the TSH level increased more in the selenium group. The final concentration of FT4 was significantly lower in those patients receiving selenium therapy $(15.8 \mathrm{pmol} / \mathrm{l}$ vs. $18.8 \mathrm{pmol} / \mathrm{l}, \mathrm{p}=0.02$ ) and the TRAb level was significantly lower in the selenium group $(2.4 \mathrm{IU} / \mathrm{l}$ vs. $5.6 \mathrm{IU} / \mathrm{l}, \mathrm{p}=0.04)$. The percentage of patients with normal TRAb level at 6 months was also significantly higher in those patients receiving selenium therapy $(19.0$ vs. $0 \%, p=0.016)$. The dose of MMI therapy in the selenium group was lower than that in the control group at 6-month follow-up $(8.0 \mathrm{mg} / \mathrm{d}$ vs. $15.0 \mathrm{mg} / \mathrm{d} ; \mathrm{p}=0.001)$ and at the last followup $(5.9 \mathrm{mg} / \mathrm{d}$ vs. $11.1 \mathrm{mg} / \mathrm{d} ; \mathrm{p}=0.009)$. Kaplan-Meier survival curve showed patients receiving selenium supplementation had a significantly higher rate of remission than controls (Log-rank test $\mathrm{p}=0.008$ ) ( 0 Fig. 3 ). The above findings suggest that selenium supplementation can enhance the effect of antithyroid drugs in patients with recurrent Graves' disease.

Previous studies have identified the beneficial effect of selenium therapy on Graves' orbitopathy, but the effect of selenium therapy on recurrent Graves' disease is still unclear [23]. There was one finished and published trial with small sample size aiming to assess the effect of selenium supplementation in patients with newly diagnosed and untreated Graves' disease [24]. Another ongoing trial assessing the effect of selenium on Grave' hyperthyroidism is the GRASS trial, which also only includes newly diagnosed and untreated Graves' disease and is not finished yet [25]. Therefore, our study is the first clinical study aim- ing to assess the efficacy of selenium therapy on recurrent Graves' disease. The present study showed an obvious effect of selenium supplementation in normalizing thyroid hormones, and normalizing serum TRAb in patients with recurrent Graves' disease. In addition, significant results were usually seen after 2 months therapy with selenium in our study. The levels of FT3 and FT4 would decrease obviously after the treatment of MMI in both the Selenium group and the control group, and most of them would become normal after about 3 month of MMI treatment. However, the selenium supplementation could increase the effect of MMI and help patients get early biochemical restoration of hyperthyroidism. At the follow-up of 3 months or 6 months, most patients in the control group also got biochemical restoration of hyperthyroidism, and thus there was no obvious difference. Therefore, the findings in our study suggest that selenium supplementation can enhance biochemical restoration of hyperthyroidism in patients with recurrent Graves' disease. In addition, the TRAb level was significantly lower in the selenium group at the last follow-up $(2.4 \mathrm{IU} / \mathrm{l}$ vs. $5.6 \mathrm{IU} / \mathrm{l}, \mathrm{p}=0.04)$ and the percentage of patients with normal TRAb level at 6 months was also significantly higher in those patients receiving selenium therapy ( 19.0 vs. $0 \%, p=0.016$ ), which suggested that selenium supplementation could be useful in reducing the autoimmunity against thyroid in patients with recurrent Graves' disease.

Increased reactive oxygen species (ROS) generation and the consequent damage of oxidative stress are involved in the development of Graves' disease [15, 16,26,27]. Restoration of euthyroidism with antithyroid drug is associated with a reversal of the biochemical abnormalities associated with oxidative stress. There is a gene-environment interaction in the fundamental process for the occurrence of Graves' disease, and selenium is an important environmental factor that may concur to the onset of Graves' disease $[2,14,28,29]$. Selenium is an essential element to thyroid hormone economy, and has important roles in the development of Graves' disease. Previous studies have shown that patients with hyperthyroidism have lower level of plasma selenium than normal controls [20-22]. Selenium deficiency proves to be a risk factor for orbitopathy or relapse in patients with Graves' disease [30,31]. Wertenbruch et al. found that the highest serum selenium level ( $>120 \mathrm{mg} / \mathrm{l}$ ) was seen in the remission group, indicating a positive effect of selenium levels on the outcome of Graves' disease [31].

Antioxidants administered together with antithyroid drugs may lead to a more rapid control of clinical manifestations and a faster normalization of thyroid function. A large randomized clinical trial has shown that antioxidant supplementation may also be beneficial for mild Graves' orbitopathy $[23,32,33]$. Vrca et al. reported a trial to assess the effect of antioxidants therapy in Graves' disease, and they used a combination of vitamin C, vitamin E, beta-carotene, and selenium [34]. They found that patients receiving antioxidants therapy together with MMI therapy recovered faster than those treated with MMI alone. The selenium therapy in patients with hyperthyroidism can increase the level of selenium in the body and thus be helpful in normalizing thyroid hormones [24]. The above effect may result from the decrease of proinflammatory cytokines caused by selenium administration $[35,36]$. The findings from our study add new evidence for the efficacy of selenium therapy in patients with recurrent Graves' disease, which also provides a promising treatment strategy for recurrent hyperthyroidism.

There are several limitations in this prospective pilot study. First, there was a greater risk of selection bias owing to the quasi-ran- 
dom in the allocation of patients. There was no statistical difference in gender, age, and the levels of serum FT4, FT3, TSH, and TRAb between the 2 groups at baseline ( $\triangle$ Table 1 ), the finding need to be validated in future randomized clinical trials. Second, the number of recruited patients was also limited. The small sample size may have led to insufficient statistical power to detect a slight effect, which is a major limitation to the reliability of the study. To get a more adequate assessment of the effect of selenium administration in patients with recurrent Graves' disease, future clinical trials are needed recruiting enough number of patients. Third, the effect of selenium supplementation may be more profound in patients with selenium deficiency, which has not been analyzed in the present study [11,13]. Fourth, there is conflicting data on the dose and form of selenium used for supplementation $[12,37,38]$. More clinical trials are still needed to find the best dose or form of selenium used for treatment in recurrent Graves' disease. In addition, owing to the pilot design of this study, we were able to assess the role of duration of selenium administration in the efficacy of treatment. Further studies are needed to assess the impact of the duration of selenium administration in the efficacy of treatment. Finally, the mechanism underling the efficacy of selenium therapy in recurrent Graves' disease has not been adequately explained, which also need further studies.

In conclusion, the findings from the study suggest that selenium supplementation can enhance the effect of antithyroid drugs in patients with recurrent Graves' disease. In addition, more randomized trials with large number of recruited participants are needed to validate the above findings.

\section{Acknowledgements}

$\nabla$

We thank all the participants in our study.

\section{Conflicts of interest}

The authors have no competing financial interests to declare.

\section{References}

1 Burch HB, Cooper DS. Management of Graves Disease: A Review. JAMA 2015; 314: 2544-2554

2 Morshed SA, Davies TF. Graves' Disease Mechanisms: The Role of Stimulating, Blocking, and Cleavage Region TSH Receptor Antibodies. Horm Metab Res 2015; 47: 727-734

3 Frank CU, Braeth S, Dietrich JW, Wanjura D, Loos U. Bridge Technology with TSH Receptor Chimera for Sensitive Direct Detection of TSH Receptor Antibodies Causing Graves' Disease: Analytical and Clinical Evaluation. Horm Metab Res 2015; 47: 880-888

4 Bartalena L. Diagnosis and management of Graves disease: a global overview. Nat Rev Endocrinol 2013; 9: 724-734

5 Anagnostis P, Adamidou F, Polyzos SA, Katergari S, Karathanasi E, Zouli C, Panagiotou A, Kita M. Predictors of long-term remission in patients with Graves' disease: a single center experience. Endocrine 2013; 44: 448-453

6 Laurberg P. Remission of Graves' disease during anti-thyroid drug therapy. Time to reconsider the mechanism? Eur J Endocrinol 2006; 155: 783-786

7 Laurberg P, Krejbjerg A, Andersen SL. Relapse following antithyroid drug therapy for Graves' hyperthyroidism. Curr Opin Endocrinol Diabetes Obes 2014; 21: 415-421

8 Park SM, Cho YY, Joung JY, Sohn SY, Kim SW, Chung JH. Excessive iodine intake does not increase the recurrence rate of Graves' disease after withdrawal of the antithyroid drug in an iodine-replete area. Eur Thyroid J 2015; 4: 36-42
9 Piantanida E, Lai A, Sassi L, Gallo D, Spreafico E, Tanda ML, Bartalena L. Outcome Prediction of Treatment of Graves' Hyperthyroidism with Antithyroid Drugs. Horm Metab Res 2015; 47: 767-772

10 Neumann S, Place RF, Krieger CC, Gershengorn MC. Future Prospects for the Treatment of Graves' Hyperthyroidism and Eye Disease. Horm Metab Res 2015; 47: 789-796

11 Duntas LH, Benvenga S. Selenium: an element for life. Endocrine 2015; 48: 756-775

12 Kohrle J. Selenium and the thyroid. Curr Opin Endocrinol Diabetes Obes 2015; 22: 392-401

13 Duntas LH. The Role of Iodine and Selenium in Autoimmune Thyroiditis. Horm Metab Res 2015; 47: 721-726

14 Tanda ML, Piantanida E, Lai A, Lombardi V, Dalle Mule I, Liparulo L, Pariani $N$, Bartalena $L$. Thyroid autoimmunity and environment. Horm Metab Res 2009; 41: 436-442

15 Marcocci C, Bartalena L. Role of oxidative stress and selenium in Graves' hyperthyroidism and orbitopathy. J Endocrinol Invest 2013; 36: $15-20$

16 Zarkovic M. The role of oxidative stress on the pathogenesis of Graves disease. J Thyroid Res 2012; 302537

17 Farrokhian A, Bahmani F, Taghizadeh M, Mirhashemi SM, Aarabi MH, Raygan F, Aghadavod E, Asemi Z. Selenium Supplementation Affects Insulin Resistance and Serum hs-CRP in Patients with Type 2 Diabetes and Coronary Heart Disease. Horm Metab Res 2016; 48: 263-268

18 Puertollano MA, Puertollano E, de Cienfuegos GA, de Pablo MA. Dietary antioxidants: immunity and host defense. Curr Top Med Chem 2011; 11: $1752-1766$

19 Marino M, Latrofa F, Menconi F, Chiovato L, Vitti P. Role of genetic and non-genetic factors in the etiology of Graves' disease. J Endocrinol Invest 2015; 38: 283-294

20 Aihara K, Nishi Y, Hatano S, Kihara M, Yoshimitsu K, Takeichi N, Ito T, Ezaki H, Usui T. Zinc, copper, manganese, and selenium metabolism in thyroid disease. Am J Clin Nutr 1984; 40: 26-35

21 Reglinski J, Smith WE, Wilson R, Halls DJ, McKillop JH, Thomson JA. Selenium in Graves' disease. Clin Chim Acta 1992; 211: 189-190

22 Bulow Pedersen I, Knudsen N, Carle A, Schomburg L, Kohrle J, Jorgensen $T$, Rasmussen $L B$, Ovesen $L$, Laurberg P. Serum selenium is low in newly diagnosed Graves' disease: a population-based study. Clin Endocrinol (Oxf) 2013; 79: 584-590

23 Marcocci C, Kahaly GJ, Krassas GE, Bartalena L, Prummel M, Stahl M, Altea MA, Nardi M, Pitz S, Boboridis K, Sivelli P, von Arx G, Mourits MP, Baldeschi L, Bencivelli $W$, Wiersinga $W$. Selenium and the course of mild Graves' orbitopathy. N Engl J Med 2011; 364: 1920-1931

24 Calissendorff J, Mikulski E, Larsen EH, Moller M. A Prospective Investigation of Graves' Disease and Selenium: Thyroid Hormones, AutoAntibodies and Self-Rated Symptoms. Eur Thyroid J 2015; 4: 93-98

25 Watt T, Cramon P, Bjorner JB, Bonnema SJ, Feldt-Rasmussen U, Gluud C, Gram J, Hansen JL, Hegedus L, Knudsen N, Bach-Mortensen P, Nolsoe R, Nygaard B, Pociot F, Skoog M, Winkel P, Rasmussen AK. Selenium supplementation for patients with Graves' hyperthyroidism (the GRASS trial): study protocol for a randomized controlled trial. Trials 2013; 14: 119

26 Banga JP, Moshkelgosha S, Berchner-Pfannschmidt U, Eckstein A. Modeling Graves' Orbitopathy in Experimental Graves' Disease. Horm Metab Res 2015; 47: 797-803

27 Li C, Tan J, Zhang G, Meng Z, Wang R, Li W, Zheng $W$. Risk factors of hyperthyroidism with hepatic function injury: a 4-year retrospective study. Horm Metab Res 2015; 47: 209-213

28 Furmaniak J, Sanders J, Nunez Miguel R, Rees Smith B. Mechanisms of Action of TSHR Autoantibodies. Horm Metab Res 2015; 47: 735-752

29 Pujol-Borrell R, Gimenez-Barcons M, Marin-Sanchez A, Colobran $R$. Genetics of Graves' Disease: Special Focus on the Role of TSHR Gene. Horm Metab Res 2015; 47: 753-766

30 Khong JJ, Goldstein RF, Sanders KM, Schneider H, Pope J, Burdon KP, Craig JE, Ebeling PR. Serum selenium status in Graves' disease with and without orbitopathy: a case-control study. Clin Endocrinol (Oxf) 2014; 80: 905-910

31 Wertenbruch T, Willenberg HS, Sagert C, Nguyen TB, Bahlo M, Feldkamp J, Groeger C, Hermsen D, Scherbaum WA, Schott M. Serum selenium levels in patients with remission and relapse of Graves' disease. Med Chem 2007; 3: 281-284

32 Marcocci C, Leo M, Altea MA. Oxidative stress in Graves' disease. Eur Thyroid J 2012; 1: 80-87

33 Salvi M, Campi I. Medical Treatment of Graves' Orbitopathy. Horm Metab Res 2015; 47: 779-788 
34 Vrca VB, Skreb F, Cepelak I, Romic Z, Mayer L. Supplementation with antioxidants in the treatment of Graves' disease; the effect on glutathione peroxidase activity and concentration of selenium. Clin Chim Acta 2004; 341: 55-63

35 Duntas LH. The evolving role of selenium in the treatment of Graves' disease and ophthalmopathy. J Thyroid Res 2012; 2012: 736161

36 Drutel A, Archambeaud F, Caron P. Selenium and the thyroid gland: more good news for clinicians. Clin Endocrinol (Oxf) 2013; 78: $155-164$
37 Wimmer I, Hartmann T, Brustbauer R, Minear G, Dam K. Selenium levels in patients with autoimmune thyroiditis and controls in lower Austria. Horm Metab Res 2014; 46: 707-709

38 Winther KH, Watt T, Bjorner JB, Cramon P, Feldt-Rasmussen U, Gluud C, Gram J, Groenvold M, Hegedus L, Knudsen N, Rasmussen AK, Bonnema SJ. The chronic autoimmune thyroiditis quality of life selenium trial (CATALYST): study protocol for a randomized controlled trial. Trials 2014; 15: 115 